The Astrophysical Journal, 624:392-401, 2005 May 1

(C) 2005. The American Astronomical Society. All rights reserved. Printed in U.S.A.

\title{
ALFVÉN WAVES AND SHOCK WAVE FORMATION AT AN X-POINT MAGNETIC FIELD CONFIGURATION
}

\author{
SimONE LANDI \\ Dipartimento di Astronomia e Scienza dello Spazio, Università degli Studi di Firenze, Largo Enrico Fermi 2, \\ 50125 Firenze, Italy; slandi@arcetri.astro.it \\ Marco Velli \\ Jet Propulsion Laboratory, California Institute of Technology, 4800 Oak Grove Drive, Pasadena, CA 91109; \\ and Dipartimento di Astronomia e Scienza dello Spazio, Università degli Studi di Firenze, Largo Enrico \\ Fermi 2, 50125 Firenze, Italy; velli@arcetri.astro.it \\ AND \\ Giorgio EinAudi \\ Dipartimento di Fisica "Enrico Fermi," Università degli Studi di Pisa, via Buonarroti 2, \\ 56127 Pisa, Italy; einaudi@df.unipi.it \\ Received 2004 May 18; accepted 2005 January 13
}

\begin{abstract}
We present MHD numerical simulations of the propagation of Alfvén waves in inhomogeneous magnetic fields whose topology consists of two-dimensional X-points threaded by a nonvanishing normal component. The coupling of the waves with the background field gradients leads to the development of fast-mode shock trains propagating normal to the average normal magnetic field. The fronts occur with a frequency matching that of the generating Alfvén wave. Energy in the original Alfvén wave packet is channeled via mode transformation to the fast waves and into the shocks, where it is dissipated at a rate independent of the Reynolds number. Such X-point shock trains might therefore play an important role in the heating of the solar corona plasma and, more generally, in cosmic particle acceleration.
\end{abstract}

Subject headings: acceleration of particles — methods: numerical — MHD — shock waves — Sun: corona

\section{INTRODUCTION}

Magnetohydrodynamic (MHD) waves are thought to play an important role in heating the solar corona and other astrophysical plasmas (Malara \& Velli 1994; Einaudi \& Velli 1994; Cranmer 2002). They are also fundamental in the acceleration and scattering of energetic particles (Vlahos 1994). In the solar corona, the classical (collisional) dissipation is very small: the Reynolds number for an active region is of the order of $10^{11}$ $10^{14}$, which means that wave energy is dissipated via small-scale structures. Since the background medium coronal magnetic field is far from homogeneous, one way to create small scales in an MHD wave is given by the interaction between the wave and the inhomogeneity. Alternatively, nonlinear effects can generate a cascade that takes energy toward small scales. In general, both phenomena occur simultaneously and can either compete with or reinforce one another (Einaudi 1995).

An X-point configuration in two dimensions threaded by an axial magnetic field is a generic feature of inhomogeneous magnetic fields both in the corona and in laboratory experiments. The study of wave propagation and magnetic reconnection at X-points has attracted much interest in connection with the investigation of the scaling of the dissipation with the Reynolds number for MHD waves (Bulanov et al. 1990; Craig \& McClymont 1993) and the more general question of the speed of the reconnection process (see Priest \& Forbes 2000 for a review).

In a plasma with negligible kinetic pressure, for perturbations that are two-dimensional in the vicinity of an X-point, i.e., for perturbations that are constant along the axial field, Alfvén waves and fast MHD waves are decoupled both in the limit of a small axial field (Bulanov \& Syrovatskii 1980) and in the limit of a large axial field (the latter limit being equivalent to incompressible MHD motion in a plane containing the X-point; Bulanov et al. 1990). In both cases wave propagation leads to the formation of current singularities at either the $\mathrm{X}$-line, for fast waves, or at the separatrix lines, for Alfvén waves (McLaughlin $\&$ Hood 2004).

Here we study the propagation of Alfvén waves in a potential magnetic field configuration with X-points for a small plasma $\beta$ (the ratio between the kinetic and magnetic pressure) and moderate values of the axial magnetic field. The main result we find is that the coupling of the shear Alfvén mode to the fast mode by the X-point magnetic geometry is extremely efficient, leading to the formation of intricate patterns of fast shock waves.

The nonlinear formation of fast waves from a propagating shear Alfvén wave is a well-known second-order effect generated by the perturbed magnetic pressure in an Alfvén wave. Such wave couplings have been observed, for example, in a compressible medium regardless of the particular value of the plasma $\beta$ or the Alfvén wave polarization (e.g., Malara et al. 1996), and, for a weak Alfvénic pulse, in propagation along an inhomogeneous, cold plasma (Nakariakov et al. 1997; Tsiklauri et al. 2001) and in the limit of both low and high $\beta$ regimes (Tsiklauri et al. 2002). In all these phenomena the magnetic pressure fluctuations are generated in the phase-mixing process (Heyvaerts \& Priest 1983), and the generated fast wave has a frequency twice that of the exciting Alfvén wave.

In this paper, we show how the coupling to the X-point geometry gradients leads to generated fast waves that have the same frequency as the Alfvén waves approaching the X-point and an amplitude that scales linearly with the amplitude of the incoming Alfvén waves. This indicates a mechanism of mode conversion 
that differs from the standard nonlinear fast wave excitation via magnetic pressure fluctuations.

In $\S 2$ we detail the model, the initial conditions, and other parameters of our numerical experiments. In particular, we focus on the use of projected characteristics in treating boundary conditions both to have essentially transparent boundaries for fluctuations leaving the simulation domain and to allow us to inject Alfvén waves from the boundaries. Although the use of projected characteristics in treating boundary conditions is widely used in numerical simulations of astrophysical plasmas, for clearness we have chosen to include its formalism in the Appendix. In $\S 3$ we describe the dynamic observed when (shear) Alfvén waves are injected from the boundaries into a domain in which the magnetic field contains an X-point topology in planes. In $\S 4$ we discuss the relevance of our results to the coronal heating problem as well as possible consequences for particle acceleration.

\section{THE NUMERICAL MODEL}

We integrate the resistive and viscous MHD equations for an isothermal plasma,

$$
\begin{gathered}
\frac{\partial \rho}{\partial t}+\nabla \cdot(\rho \boldsymbol{v})=0 \\
\frac{\partial \boldsymbol{v}}{\partial t}+\boldsymbol{v} \cdot \nabla \boldsymbol{v}=-\frac{c^{2}}{\rho} \nabla \rho+(\nabla \times \boldsymbol{B}) \times \boldsymbol{B}+\frac{1}{R_{\nu}} \nabla^{2} \boldsymbol{v} \\
\frac{\partial \boldsymbol{B}}{\partial t}=\nabla \times(\boldsymbol{v} \times \boldsymbol{B})+\frac{1}{R_{\eta}} \nabla^{2} \boldsymbol{B}
\end{gathered}
$$

where $\boldsymbol{v}$ is the fluid velocity, $\boldsymbol{B}$ is the magnetic field, $\rho$ is the density, and $c^{2}$ is the isothermal sound speed. The equations are expressed in dimensionless units, defined by a characteristic length $L_{0}$, a characteristic density $\rho_{0}$, and a characteristic magnetic field strength $B_{0}$. The nondimensional velocity field as well the isothermal sound speed are therefore in units of the Alfvén speed $v_{\mathrm{A}}=\left[B_{0}^{2} /\left(4 \pi \rho_{0}\right)\right]^{1 / 2}$, while time is in units of the Alfvén crossing time $t_{0}=L_{0} / v_{\mathrm{A}}$. The viscous and resistive dissipative coefficients thus contain the Reynolds number $R_{\nu}=L_{0} v_{\mathrm{A}} / \nu_{0}$ and the Lundquist number $R_{\eta}=L_{0} v_{\mathrm{A}} / \eta_{0}$, where $\nu_{0}$ and $\eta_{0}$ are the kinematic viscosity and the magnetic diffusivity, respectively. From here, we assume $R_{\eta}=R_{\nu}$.

We substitute the energy equation with the isothermal approximation (and consider the temperature to be spatially uniform), since we are focusing our attention on the dynamical coupling of Alfvén waves with the surrounding inhomogeneous magnetic field and not, in this paper, on quantifying plasma heating and changes in the thermodynamic properties, which would require additional hypotheses on the density, structure of the plasma, and conductive and radiative losses both in the volume and at the boundaries. On the other hand, the high coronal temperature implies large conductivities and rapid damping of local temperature fluctuations.

The above equations are solved in 2.5 dimensions, i.e., assuming three-dimensional vector fields but an ignorable coordinate (say, $z$ ), so that fields only depend on variables in planes orthogonal to the mean field. The magnetic field can be written in terms of the $z$-component of the vector potential $\phi(x, y)$ as

$$
\boldsymbol{B}=\left(\frac{\partial \phi}{\partial y}+B_{x 0}\right) \hat{x}-\left(\frac{\partial \phi}{\partial x}-B_{y 0}\right) \hat{y}+B_{z} \hat{z}
$$

where $B_{x 0}$ and $B_{y 0}$ are constants.
Equations (1), (2), and (3), with $\boldsymbol{B}$ described by equation (4), are integrated in a rectangular box of dimensions $L_{x}=L_{y}=2 \pi$. In the first set of simulations shown here we have assumed periodicity in one dimension $(y)$, and spatial integration is performed by spectral methods. In the $x$-direction no assumption is made about periodicity of the system, and appropriate nonreflecting boundary conditions are imposed, specifying a flux of Alfvén waves into the X-point at some distance. The spatial integration is performed by the use of compact difference schemes (Lele 1992). In the second set of simulations the periodic boundary condition along $y$ is relaxed. The spatial integration is then performed using the same method used for the $x$-direction with nonreflecting boundary conditions, as specified below. The time integration is performed with a third-order Runge-Kutta method.

A drawback of periodicity along the $y$-axis is that one necessarily has to deal with multiple X-points. A simple appropriate current-free magnetic configuration is determined by the potential

$$
\begin{aligned}
\phi & =\frac{1}{2 \sinh (\pi)} \sinh (x-\pi) \sin (y), \\
B_{z} & =B_{z 0} .
\end{aligned}
$$

The magnitude of the constant field in the $z$-direction is chosen so that all three field components have similar rms magnitudes at the boundaries, and small constant field components in the $x$ - and $y$-directions are considered to avoid structurally unstable field configurations in which the multiple X-points are connected via separatrices (Bulanov et al. 1999), so $B_{x 0}=B_{y 0}=0.01$ and $B_{z 0}=0.5$. The magnetic configuration is immersed in a uniform plasma of density $\rho=1$.

In the $x$-direction, boundary conditions are assigned by the use of projected characteristics of the MHD equations (Thompson 1987; Vanajakshi et al. 1989; Poinsot \& Lele 1992; Roe \& Balsara 1996). This allows essentially transparent boundary conditions for fluctuations leaving the domain in the $x$-direction, while retaining control over the type of fluctuations input into the numerical domain. The projected characteristics have been successfully used to simulate acoustic wave injection in a nonmagnetized wind (Grappin et al. 1997; Del Zanna et al. 1998), Alfvén waves in a magnetized wind (Grappin et al. 2000; Einaudi et al. 2001), and large-amplitude Alfvén waves for studies of parametric decay (Del Zanna et al. 2001). In the initial magnetic configuration described above we have chosen to force Alfvén waves from both boundaries, $x=0$ and $x=2 \pi$. The injection of Alfvén waves from the boundaries using the characteristic formalism can be done by introducing a triggering function (see eq. [A21] in the Appendix) in the characteristic Alfvén wave (eq. [A7]). For the simulations presented here the forcing has been chosen to be of the form

$$
f(y, t)=\alpha g(y)\left(\frac{t^{2}}{t^{2}+1}\right) \cos (\omega t) .
$$

The Alfvén wave is then characterized by a maximum intensity $\alpha$ and a given frequency $\omega$. The factor $t^{2} /\left(1+t^{2}\right)$ is a ramp function, which ensures a smooth injection of the Alfvén wave at small times. The wave front along the periodic direction has been chosen to vanish outside the region in which the $x$-component of the initial magnetic field is negative in $x=0$ and positive in $x=2 \pi$. This means that we have chosen to force waves only over the central half of the domain, i.e., only one X-point, with 

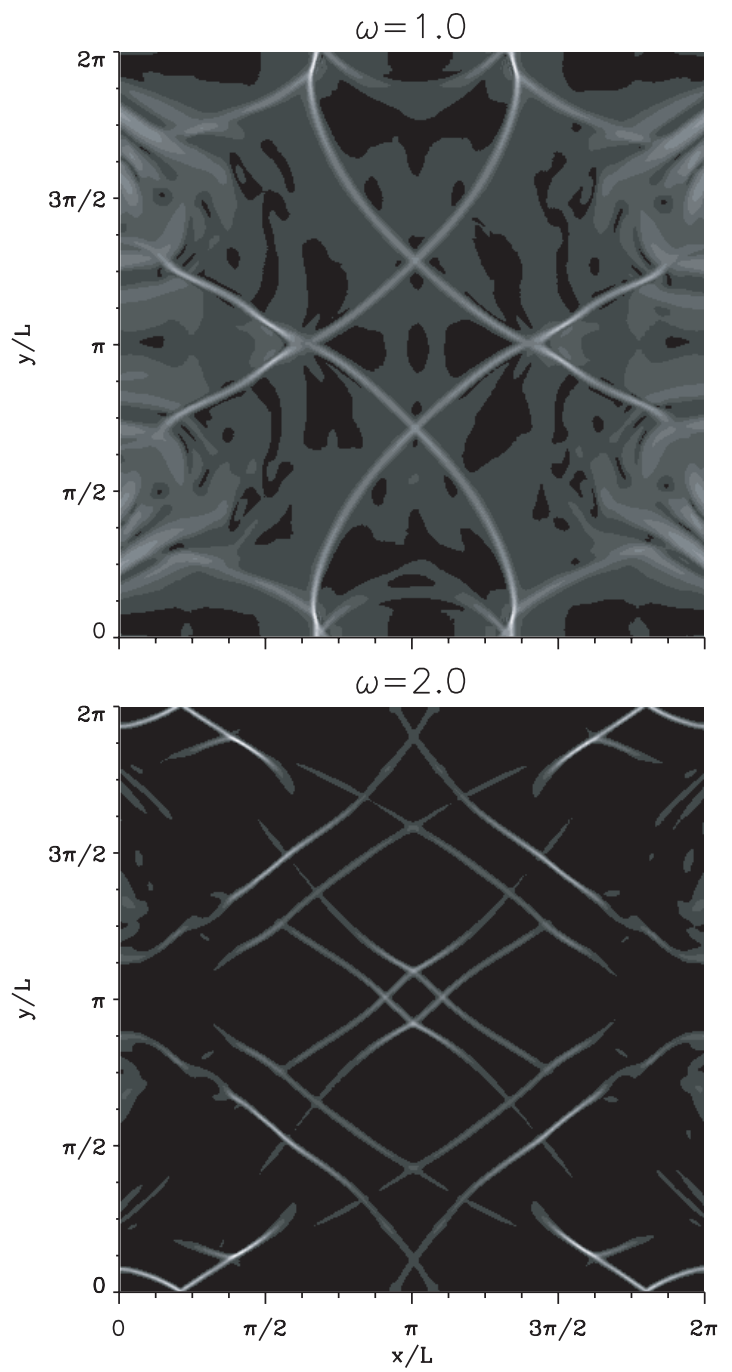
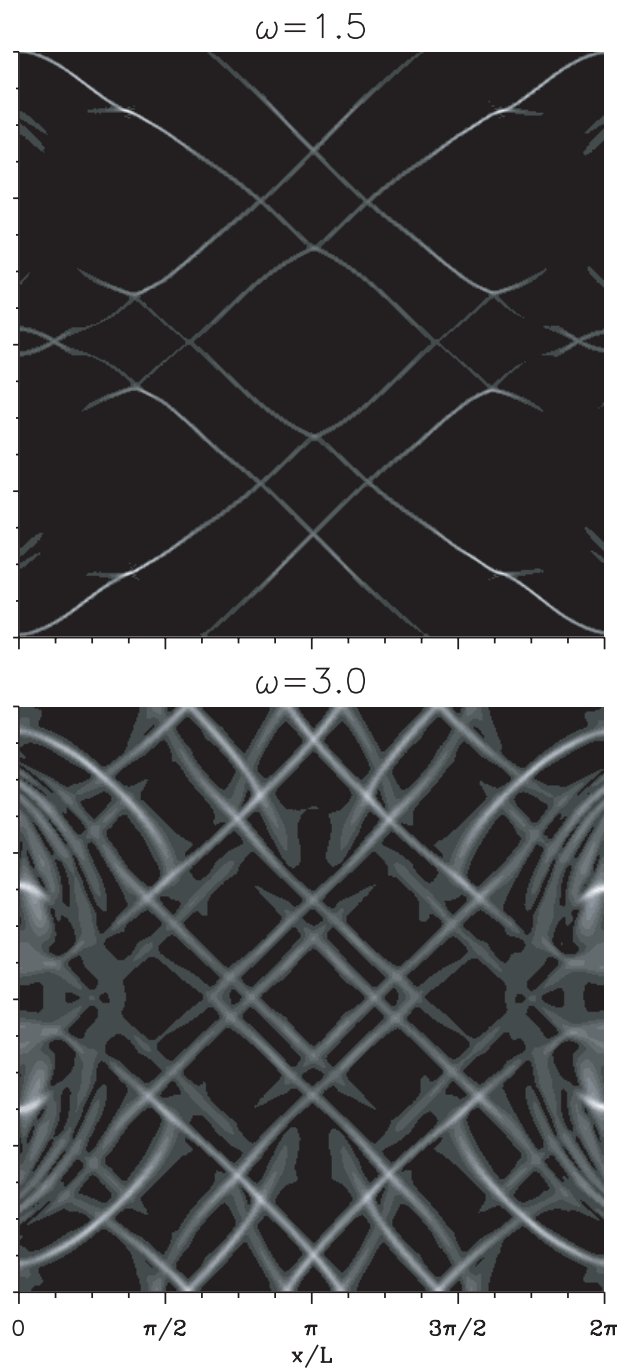

FIG. 1.-Current intensities for simulations differing in the frequency $\omega$ of the input Alfvén wave. All the simulations have $\alpha=0.5$ and $R_{\eta}=1000$.

a profile that tends to vanish smoothly where $B_{x}$ changes sign. We have used different kinds of envelopes, but no particular differences in the behavior of the system evolution have been observed. Here we show results obtained using the profile

$$
g(y)=\sin (y) \cos ^{2}(y)
$$

The fluctuations injected via the Alfvén characteristic are polarized in a complex way because of the presence of a magnetic field that, in principle, is not normal to the boundary. However, it can be shown that if no magnetoacoustic wave is injected, the Alfvén wave is characterized by a constant modulus for the perpendicular component of the magnetic field (Grappin et al. 2000), so that no second-order magnetic pressure perturbations, which would immediately lead to slow and fast modes, are driven into the domain.

\section{RESULTS}

\subsection{Alfvén Waves in Multiple X-Points}

In the simulations shown here we assume a periodic condition along the $y$-direction. As already pointed out, this means that we are dealing with multiple X-points. We have adopted a (nondimensional) temperature $T_{0}=0.075$, so that the plasma $\beta$ varies from $\beta=0.3$ near the boundary to $\beta=0.6$ around the X-points.

Different simulations have been performed varying both the frequency of the injected Alfvén wave $\omega$ and its amplitude $\alpha$, with Lundquist numbers $R_{\eta}=200,500$, and 1000 and correspondingly different resolutions $\left(n_{x}=n_{y}=128,256\right.$, and 512, respectively). Figure 1 shows the current density intensity for a set of simulations once the stationary regime has been reached on average. The simulations differ in the frequency of the injected Alfvén waves: $\omega=1.0,1.5,2.0$, and 3.0. For all simulations the amplitude of the wave is $\alpha=0.5$, i.e., of the same order as the equilibrium magnetic field configuration, and the Reynolds number is $R_{\eta}=500$. The coupling of the injected Alfvén waves with the surrounding magnetic field leads to the formation of thin current concentrations, whose number increases with the frequency of the ingoing Alfvén wave. These current singularities are associated with the propagation of fastmode shock waves, across which the magnetic field is not continuous. This can be shown by plotting fluctuations of $B_{z}, \rho$, and $v_{y}$ as a function of $y$ and observing their correlations. This is shown in Figure 2 for the simulation with $\omega=1.5$ and $\alpha=0.5$, for $x=1.25 \pi$, where the structures are well developed. With the arrows we have marked the direction of propagation of the two structures. 


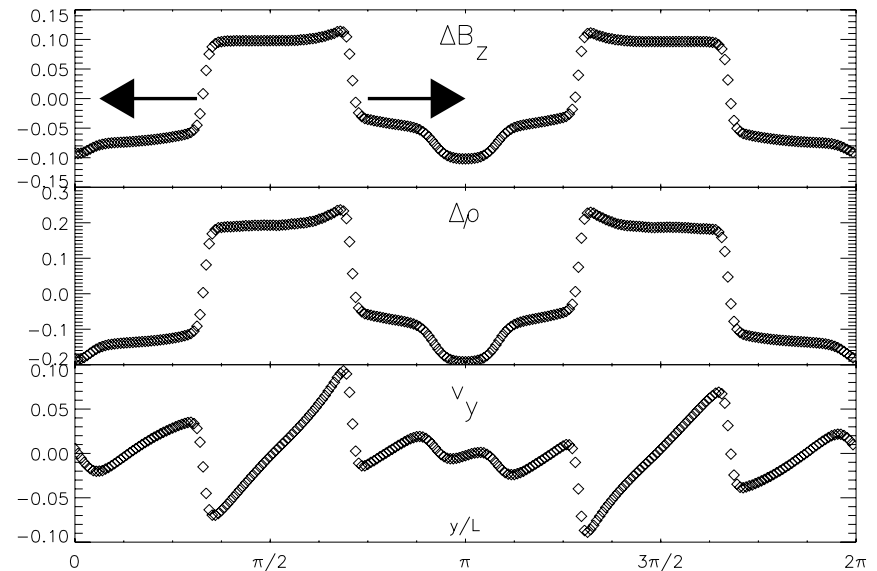

Fig. 2.-From top to bottom: Magnetic field fluctuations $\Delta B_{z}$, density fluctuations $\Delta \rho$, and $v_{y}$ profiles as a function of $y$ at a given time for the case $\omega=1.5$. The cut has been taken in $x=1.25 \pi$. The structures are propagating in the direction marked by the arrows.

From Figure 2 we recognize the positive correlation between the density and the $z$-component of the magnetic field, characteristic of fast-mode waves. These waves are propagating normal to the ambient magnetic field, which in the middle of the simulation domain is dominated by $B_{z}$. The wave front is moving with a velocity corresponding to the local fastmode speed. The gas is compressed crossing the jump, and the fluid velocity just before the jumps is greater than the fastmode speed in the wave front reference frame. Careful analysis shows that the Rankine-Hugoniot jump conditions in the limit of an isothermal gas are verified. For a wave steepened into shock its wavelength $\lambda_{f}$ is given by the distance between two jumps propagating in the same direction, and, using the approximate WKB dispersion relation $\omega_{f}=2 \pi\left(a^{2}+c^{2}\right)^{1 / 2} / \lambda_{f}$ (valid for fast waves propagating normally to the ambient magnetic field, $a^{2}$ being the magnitude of the Alfvén wave), we can estimate the frequency of the excited fast mode. This frequency corresponds to those of Alfvén waves injected from the boundaries.

In Figure 3 we show the current dissipation rate averaged in the whole domain for the simulations shown in Figure 1. In each panel of Figure 3 we compare the dissipation between simulations having the same Alfvén wave frequency and amplitude but a different Reynolds number. Because the injected Alfvén mode is efficiently converted into fast-mode waves steepening into shocks, the magnetic energy is mainly dissipated in the thin current structures carried by the shock waves. As a consequence, the dissipation is almost independent of the Reynolds number, and using this fact, we can find a relationship between $R_{\eta}$ and the width $l$ of the current sheet formed by the sharp $B_{z}$ gradient across the shock. Using the total energy dissipation rate $W_{\eta}=j^{2} l L / R_{\eta}$ in one current sheet of length $L$ and taking into account that $j \simeq \Delta B_{z} / l$, with $\Delta B_{z}$ depending only on the RankineHugoniot jump conditions, we obtain $l \propto 1 / R_{\eta}$. This relationship between the current sheet width and the Reynolds number is verified in our simulations.

An important question is how the formation of the fast shock waves depends on the amplitude $\alpha$ of the Alfvén waves injected at the boundaries. In the top panel of Figure 4 we show the ohmic dissipation rate averaged in the whole domain as a function of time for simulations with $\omega=1.0$ and $R_{\eta}=500$, where the amplitude of the Alfvén wave varies from $\alpha=0.5$ to 0.1 . The current dissipation has been rescaled, dividing it by $\alpha^{2}$. All the profiles are virtually superposed. This means that the intensity of the dissipation scales as the square of the amplitude of the
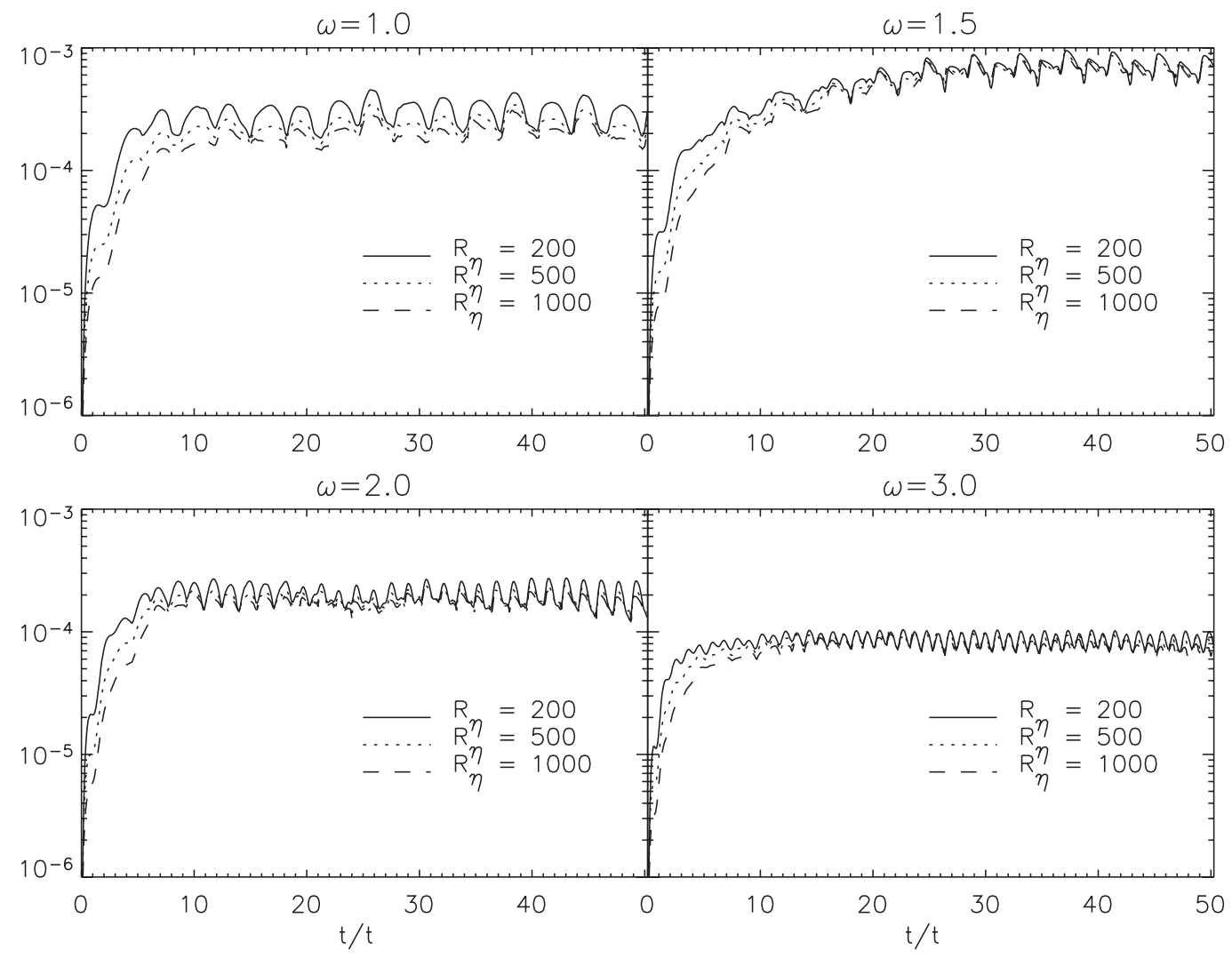

FIG. 3.-Ohmic dissipation as a function of time. For each simulation shown in Fig. 1 we have plotted the ohmic dissipation using different Reynolds numbers: $R_{\eta}=200,500$, and 1000 . 


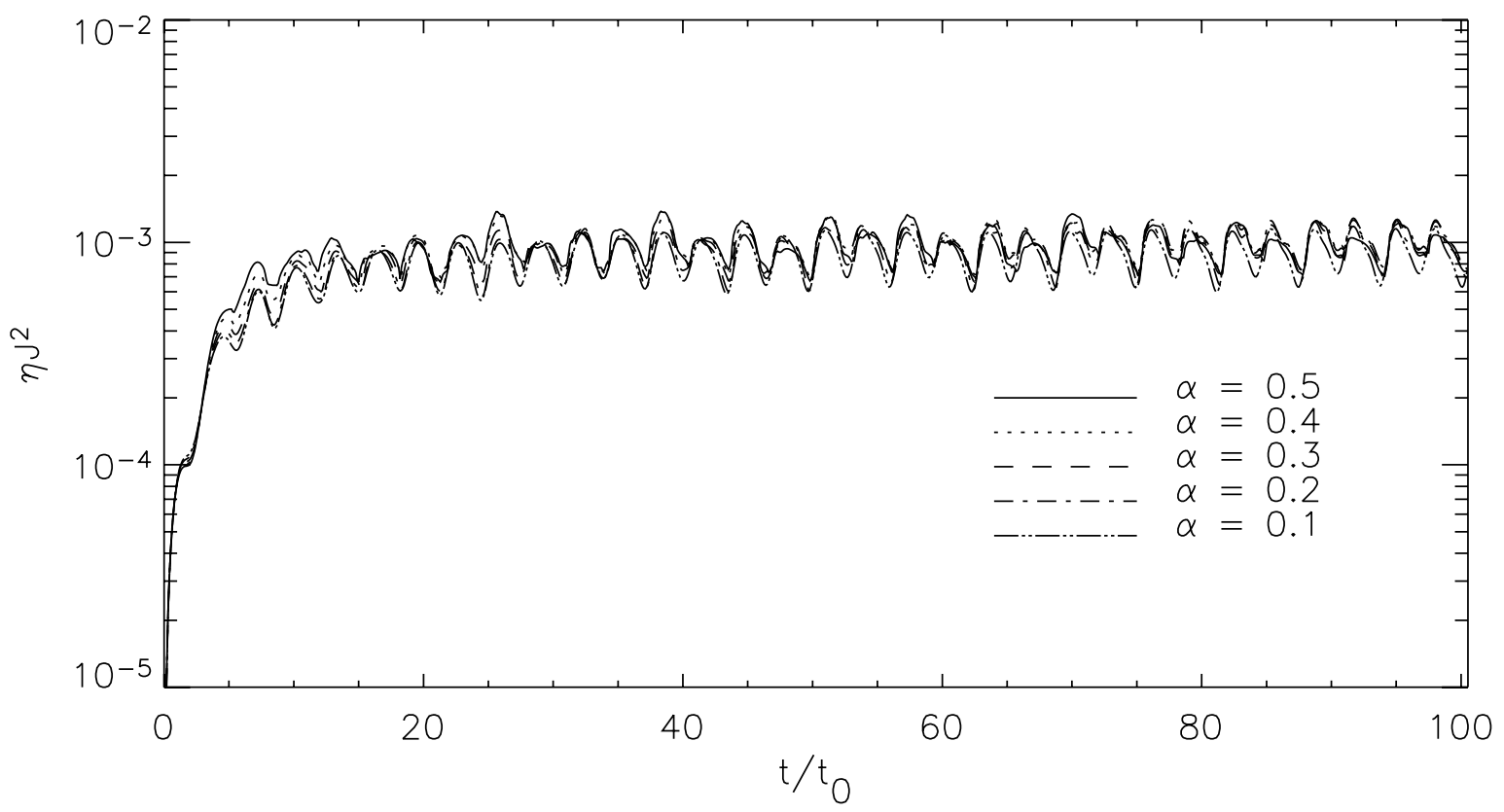

Fig. 4.-Ohmic dissipation rate for simulations with $\omega=1$ and $R_{\eta}=500$ with different values of the amplitude $\alpha$ of the Alfvén waves coming from the open boundaries. The dissipation for each simulation has been rescaled, dividing it by $\alpha^{2}$.
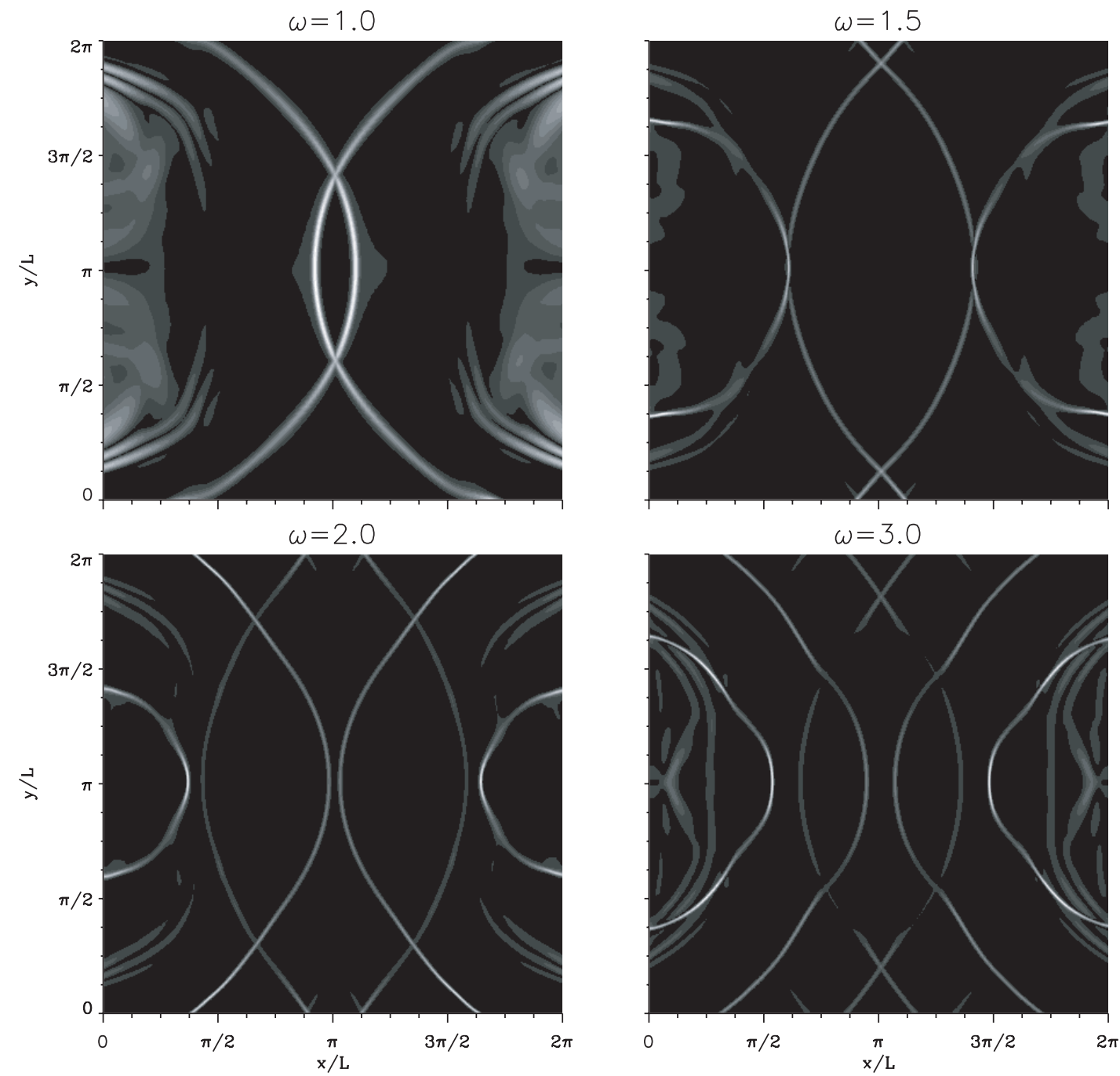

Fig. 5.-Current intensities for simulations differing in the frequency $\omega$ of the input Alfvén wave. All the simulations have $\alpha=0.5$ and $R_{\eta}=1000$. Simulations here are performed using open boundary conditions from both directions. 

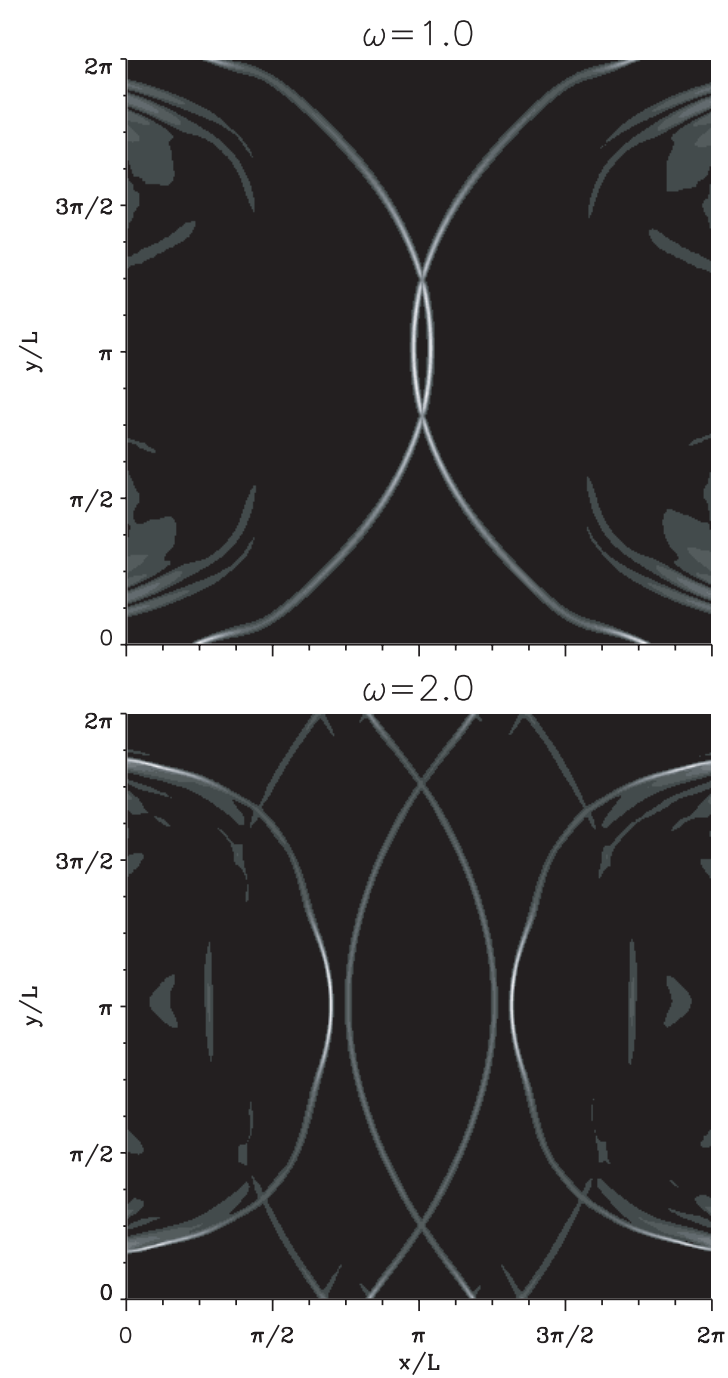

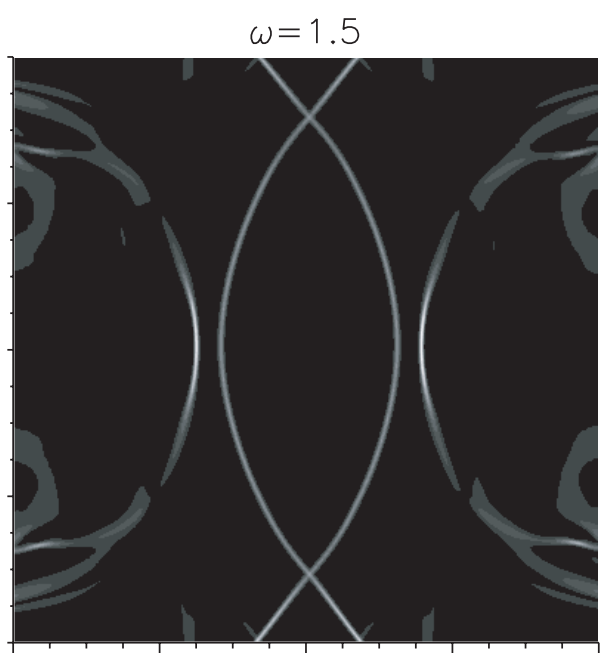

$\omega=3.0$

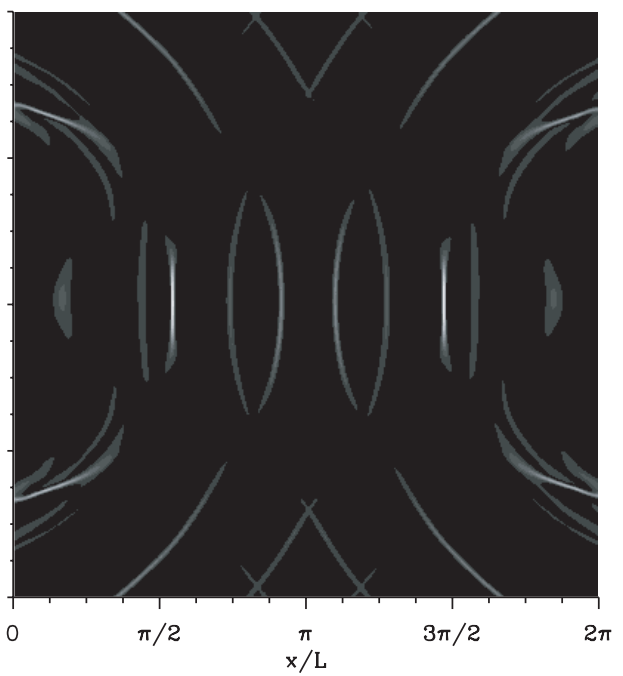

FIG. 6. - Current intensities for simulations differing in the frequency $\omega$ of the input Alfvén wave. All the simulations have $\alpha=0.5$ and $R_{\eta}=500$. In this case simulations are performed with $\beta$ that is 1 order of magnitude less than the previous one.

injected Alfvén waves and that the equilibrium between the net energy flux coming from the boundaries and the dissipation is reached independently of $\alpha$.

This behavior can be explained in terms of a linear coupling between the Alfvén wave and the fast-mode wave. Because the ohmic dissipation is mainly due to the $B_{z}$ jump at the shock front, we have $j \approx \Delta B_{z} / l$, so that when the energy equilibrium is reached $\Delta B_{z} \propto \alpha$. Now, if we have a linear coupling between the Alfvén wave and the magnetoacoustic wave caused by a resonant process, the amplitude of the magnetoacoustic wave should depend linearly both on the amplitude of the injected Alfvén wave and on the time, i.e., $\Delta B_{z}(t) \simeq \alpha t$. Thus, the time needed to reach the asymptotic value will be independent of the amplitude of the Alfvén waves coming from the boundaries.

\subsection{Alfvén Waves in a Single X-Point}

How much does the presence of multiple X-points contribute to the strength of the shock waves? The coherence of the fast mode reentering the simulation domain along the periodic direction could add to the effectiveness of the shock-forming mechanism, and we have therefore carried out simulations in which the periodicity assumption along the $y$-direction is relaxed. In this case boundary conditions along $y$ are assigned, as in the $x$-direction, with the use of projected boundary conditions. Along the $y$-direction characteristics are used only for the purpose of nonreflecting boundary conditions, so no waves are injected from the boundaries at $y=0$ and $y=2 \pi$. We have adopted the same values for density, temperature, and magnetic field strength used in $\S 3.1$.

In Figure 5 we show current density intensities for a set of simulations in which Alfvén waves are, as in $\S 3.1$, characterized by $\alpha=0.5$ and $\omega=1.0,1.5,2.0$, and 3 . The resolution used here is $n_{x}=n_{y}=512$, corresponding to magnetic and kinetic Reynolds numbers equal to $R_{\nu}=R_{\eta}=1000$. As in the case in which periodic boundary conditions are present, we recognize the presence of thin current concentrations that correspond to the propagation of fast shock waves. Their number increases with the frequency of the injected Alfvén wave, and they also propagate at the local fast wave speed. As for the simulations shown previously, we have studied the effects of varying the Reynolds number as well as the amplitude of the Alfvén wave. As before, we observe that the dissipation is dominated by the fast shock waves and does not depend on the Reynolds number. The dissipation intensity scales as $\alpha^{2}$, and equilibrium between dissipation and energy flux coming from the boundaries is reached independently of $\alpha$. The only difference we observe is that now the propagation of the fast shock waves yields a less 
intricate pattern than that where periodic boundary conditions are used. The reason is simply that waves reaching the boundaries are now not allowed to reenter the simulation domain.

The choice of a relatively high plasma $\beta$ (at least for solar coronal applications) can also play a role in the coupling and formation of fast wave shock trains. For an active region with a typical temperature of about $(1-2) \times 10^{6} \mathrm{~K}$, a density of $10^{8}-$ $10^{9} \mathrm{~cm}^{-3}$, and a magnetic field strength of about $10 \mathrm{G}$, the plasma $\beta$ is no more than 0.07 . In Figure 6 we show results obtained by adopting $c^{2}=0.0075,1$ order of magnitude less than that previously used, while all the other parameters retain the same values as for the simulations shown in Figure 5. This value corresponds to a plasma $\beta$ that varies between 0.03 near the boundary and 0.06 around the X-point. The figure shows that the fast-mode shocks still develop, so that the plasma $\beta$ does not play an appreciable role, at least in the $\beta<1$ regime.

\section{CONCLUSION}

We have presented numerical simulations of the propagation of large-amplitude Alfvén waves in the presence of X-point magnetic field topologies. We have found that the X-point field gradients lead to mode conversion into fast-mode shocks. Our results seem to indicate that this route to dissipation might be as important as the direct dissipation of the waves (Bulanov et al. 1990; Craig \& McClymont 1993, 1997; McLaughlin \& Hood 2004), since dissipation via shocks does not depend on the Reynolds number, and therefore the whole problem related to the timescales of dissipation in the question of coronal heating is bypassed in this process.

The results presented here should be seen as preliminary results. Several aspects should be further investigated in order to estimate the efficiency of this mechanism in the coronal heating.

In the corona, typical values for the Alfvén speed are about $2 \times 10^{8} \mathrm{~cm} \mathrm{~s}^{-1}$; assuming a typical length scale of $L=10^{9} \mathrm{~cm}$ for a coronal loop, the characteristic time is about $5 \mathrm{~s}$, so that the period for the lower frequency Alfvén wave used here $(\omega=1)$ is about $30 \mathrm{~s}$. This means that we are using a rather high frequency but amplitudes that are comparable in value with the initial magnetic configuration. The reason for this choice comes essentially from numerical constraints. The fast waves generated by the coupling of the injected Alfvén waves steepen into shock waves over a timescale that depends on the wavelength and amplitude of the wave, namely,

$$
\tau_{s}=\frac{\lambda_{f}}{4 \delta v}=\frac{2 \pi f}{\omega \delta v},
$$

where $\delta v$ is the amplitude of the velocity fluctuation along the propagation direction. During this time, the fast wave propagates over a length scale

$$
L_{s}=\tau_{s} f=2 \pi \frac{f^{2}}{\omega \delta v},
$$

which, in units of the total length of the simulation domain $L=2 \pi$, is

$$
\frac{L_{s}}{L}=\frac{f^{2}}{\omega \delta v} .
$$

We have seen that the coupling between the Alfvén and fast magnetoacoustic mode is linear and hence that $\omega$ is the Alfvén wave frequency and $\delta v$ is linearly proportional to its amplitude $\alpha$. From this equation it is clear that, assuming a fast wave phase velocity of the order of unity, in order to observe shock wave formation in the simulation domain we cannot choose an arbitrary low amplitude and frequency of the injected Alfvén wave.

Another constraint comes from the mandatory use of unrealistic, low values for the magnetic and kinetic Reynolds numbers. The shock wave formation time $\tau_{s}$ must be much smaller than the dissipative timescale, which turns out be

$$
\tau_{\eta}=\frac{R_{\eta}}{k^{2}}=\frac{R_{\eta} \omega^{2}}{f^{2}},
$$

and then

$$
\frac{\tau_{s}}{\tau_{\eta}} \sim \frac{1}{\delta v R_{\eta}} .
$$

To have $\tau_{s} \gg \tau_{\eta}$, with the typical values used here for the Reynolds number, the amplitude (in units of the Alfvén speed) cannot be lower than a few percent. This value corresponds to the amplitude of the velocity fluctuations observed when $\alpha=$ 0.1 (the lowest Alfvén wave amplitude used here).

On the other hand, energy stored by slow motions in the photosphere might be released in bursts in the corona, where as a consequence large-amplitude and higher frequency Alfvén waves can be generated (Longcope \& Sudan 1992a, 1992b; Axford et al. 1999). Recent observations with the SOHO and TRACE spacecrafts have shown transverse loop oscillations produced by flares in a system of magnetic loops (Aschwanden et al. 1999; Nakariakov et al. 1999), which could impulsively generate high-amplitude Alfvén waves (Del Zanna et al. 2005) with periods spanning a large interval (Aschwanden et al. 2002), from around 2 to 10 minutes. Moreover, the observed range of periods seems to be restricted only by instrumental limits (Aschwanden et al. 2002). Large-amplitude and high-frequency Alfvén waves can then interact with the inhomogeneous magnetic field corona, where two-dimensional null points are formed, as, for example, between interacting loops. The coupling with the inhomogeneous magnetic field could create compressive waves in the form of fast magnetoacoustic waves, which, steepening into shocks, are able to quickly dissipate their energy. We stress, however, that the model presented is very simple and should be viewed as an experiment of a basic process of potential interest that has never been described in detail before, rather than as a global model for coronal heating.

A final limitation of our results concerns the use of a monochromatic driver, while a spectrum of waves of varying frequency and incoherent phases would be more likely to occur in a coronal situation. An incoherent time-dependent driver might change the timescale for mode conversion. With these caveats, the process we have described should have important implications also in the problem of particle acceleration in astrophysical bodies. It is well known that particles are accelerated in shock waves by either the drift mechanism or the diffusive mechanism (Jones \& Ellison 1991). Anastasiadis \& Vlahos (1991, 1994) and Vlahos (1994) have shown that an ensemble of quasiperpendicular propagating shock waves in an active region can be an efficient particle accelerator. The resulting energy distribution, as well as the maximum kinetic energy the particles can reach, depends on the number of shocks. Although in their model shock waves in the active region are assumed to be mainly produced by the intense local heating caused by reconnection or when the slow shocks propagate away from the reconnection region, the presence of patterns of shock waves 
propagating from local X-point fields could enhance particle scattering and increase the efficiency of particle acceleration (Vlahos 1994). Test simulations to probe this process are currently being carried out.

In this work Simone Landi has been supported by the Italian Ministry of University and Research (MIUR) under grant
COFIN2002 (code 2002-02-5872) and by the Research Training Network (RTN) Theory, Observation and Simulation of Turbulence in Space Plasmas, funded by the European Commission (contract HPRN-CT-2001-00310). We are grateful to the anonymous referee for his useful suggestions and comments that improved the paper. We are also grateful to L. Del Zanna for his useful comments and to A. Anastasiadis and L. Vlahos for stimulating discussions.

\section{APPENDIX}

\section{MHD EQUATIONS IN CHARACTERISTIC FORM}

The ideal MHD equations are a set of hyperbolic partial differential equations that can be written formally as

$$
\frac{\partial \boldsymbol{U}}{\partial t}+\boldsymbol{A} \frac{\partial \boldsymbol{U}}{\partial x}+\boldsymbol{C} \frac{\partial \boldsymbol{U}}{\partial y}+\boldsymbol{D}=0 .
$$

Here $\boldsymbol{U}$ is the vector of the primitive variables, and the matrix $\boldsymbol{D}$ represents terms that do not contain any derivative, as gravitation or curvature terms. Equation (A1) is written assuming $z$ as an ignorable coordinate. The projected characteristics along the $x$-direction for the MHD equations are obtained by diagonalizing the matrix $\boldsymbol{A}$,

$$
\boldsymbol{r}_{i}^{T} \frac{\partial \boldsymbol{U}}{\partial t}+\lambda_{i} \boldsymbol{r}_{i}^{T} \frac{\partial \boldsymbol{U}}{\partial x}+\boldsymbol{r}_{i}^{T} \boldsymbol{C} \frac{\partial \boldsymbol{U}}{\partial y}+\boldsymbol{r}_{i}^{T} \boldsymbol{D}=0
$$

leading to a set of equations exactly equivalent to the original equations (A1),

$$
\frac{\partial \boldsymbol{U}}{\partial t}+\boldsymbol{S}^{-1} \mathcal{L}+\boldsymbol{C} \frac{\partial \boldsymbol{U}}{\partial y}+\boldsymbol{D}=0 .
$$

Here $\boldsymbol{S}^{-1}$ is the matrix whose columns are the right eigenvectors $\boldsymbol{r}_{i}$ corresponding to the eigenvalue $\lambda_{i}$. The components of the vector

$$
\mathcal{L}=\boldsymbol{\Lambda} S \frac{\partial \boldsymbol{U}}{\partial x}
$$

are the projected characteristics along the $x$-direction, where $\boldsymbol{\Lambda}=\boldsymbol{S A} \boldsymbol{S}^{-1}=\operatorname{diag}\left(\lambda_{i}\right)$ is the diagonal matrix containing the eigenvalues $\lambda_{i}$. For the MHD equations the set $\boldsymbol{U}$ of the primitive variables is

$$
\boldsymbol{U}=\left(\rho, v_{x}, v_{y}, v_{z}, B_{y}, B_{z}, T\right)^{T}
$$

Here $B_{x}$ is not present because its evolution equation does not show any dependence on the $x$-derivatives. Its evolution can be simply obtained by the use of the divergence-free condition. As is well known, the MHD equations admit seven different kinds of wave, whose speeds are $v_{x}+f, v_{x}+s, v_{x}+a_{x}, v_{x}, v_{x}-a_{x}, v_{x}-s$, and $v_{x}-f$, where $f$ and $s$ are the fast and slow magnetoacoustic velocities, respectively, and $a_{x}$ is the Alfvén wave along the $x$-direction. The wave speed $v_{x}$ corresponds to the entropy mode that is just advected. The characteristics corresponding to each wave are

$$
\begin{aligned}
& \mathcal{L}_{f}^{+}=\left(v_{x}+f\right)\left\{\alpha_{1}\left[\frac{1}{f}\left(\frac{T}{\rho} \frac{\partial \rho}{\partial x}+\frac{\partial T}{\partial x}\right)+\frac{\partial v_{x}}{\partial x}\right]+\alpha_{2}\left[\frac{1}{\sqrt{4 \pi \rho}}\left(\frac{a_{z}}{a_{\perp}} \frac{\partial B_{z}}{\partial x}+\frac{a_{y}}{a_{\perp}} \frac{\partial B_{y}}{\partial x}\right)-\frac{a_{x}}{f}\left(\frac{a_{z}}{a_{\perp}} \frac{\partial v_{z}}{\partial x}+\frac{a_{y}}{a_{\perp}} \frac{\partial v_{y}}{\partial x}\right)\right]\right\} \\
& \mathcal{L}_{a}^{+}=\left(v_{x}+\xi a_{x}\right)\left[\frac{a_{y}}{a_{\perp}} \frac{\partial v_{z}}{\partial x}-\frac{a_{z}}{a_{\perp}} \frac{\partial v_{y}}{\partial x}-\frac{\xi}{\sqrt{4 \pi \rho}}\left(\frac{a_{y}}{a_{\perp}} \frac{\partial B_{z}}{\partial x}-\frac{a_{z}}{a_{\perp}} \frac{\partial B_{y}}{\partial x}\right)\right], \\
& \mathcal{L}_{s}^{+}=\left(v_{x}+s\right)\left\{-\alpha_{2}\left[\frac{1}{c}\left(\frac{T}{\rho} \frac{\partial \rho}{\partial x}+\frac{\partial T}{\partial x}\right)+\xi \frac{a_{x}}{f} \frac{\partial v_{x}}{\partial x}\right]+\alpha_{1}\left[\frac{c}{f \sqrt{4 \pi \rho}}\left(\frac{a_{z}}{a_{\perp}} \frac{\partial B_{z}}{\partial x}+\frac{a_{y}}{a_{\perp}} \frac{\partial B_{y}}{\partial x}\right)-\xi\left(\frac{a_{z}}{a_{\perp}} \frac{\partial v_{z}}{\partial x}+\frac{a_{y}}{a_{\perp}} \frac{\partial v_{y}}{\partial x}\right)\right]\right\} \\
& \mathcal{L}_{0}=v_{x}\left[-\frac{c(\gamma-1)}{\gamma \rho} \frac{\partial \rho}{\partial x}+\frac{c}{\gamma T} \frac{\partial T}{\partial x}\right], \\
& \mathcal{L}_{s}^{-}=\left(v_{x}-s\right)\left\{-\alpha_{2}\left[\frac{1}{c}\left(\frac{T}{\rho} \frac{\partial \rho}{\partial x}+\frac{\partial T}{\partial x}\right)-\xi \frac{a_{x}}{f} \frac{\partial v_{x}}{\partial x}\right]+\alpha_{1}\left[\frac{c}{f \sqrt{4 \pi \rho}}\left(\frac{a_{z}}{a_{\perp}} \frac{\partial B_{z}}{\partial x}+\frac{a_{y}}{a_{\perp}} \frac{\partial B_{y}}{\partial x}\right)+\xi\left(\frac{a_{z}}{a_{\perp}} \frac{\partial v_{z}}{\partial x}+\frac{a_{y}}{a_{\perp}} \frac{\partial v_{y}}{\partial x}\right)\right]\right\} \\
& \mathcal{L}_{a}^{-}=\left(v_{x}-\xi a_{x}\right)\left[\frac{a_{y}}{a_{\perp}} \frac{\partial v_{z}}{\partial x}-\frac{a_{z}}{a_{\perp}} \frac{\partial v_{y}}{\partial x}+\frac{\xi}{\sqrt{4 \pi \rho}}\left(\frac{a_{y}}{a_{\perp}} \frac{\partial B_{z}}{\partial x}-\frac{a_{z}}{a_{\perp}} \frac{\partial B_{y}}{\partial x}\right)\right], \\
& \mathcal{L}_{f}^{-}=\left(v_{x}-f\right)\left\{\alpha_{1}\left[\frac{1}{f}\left(\frac{T}{\rho} \frac{\partial \rho}{\partial x}+\frac{\partial T}{\partial x}\right)-\frac{\partial v_{x}}{\partial x}\right]+\alpha_{2}\left[\frac{1}{\sqrt{4 \pi \rho}}\left(\frac{a_{z}}{a_{\perp}} \frac{\partial B_{z}}{\partial x}+\frac{a_{y}}{a_{\perp}} \frac{\partial B_{y}}{\partial x}\right)+\frac{a_{x}}{f}\left(\frac{a_{z}}{a_{\perp}} \frac{\partial v_{z}}{\partial x}+\frac{a_{y}}{a_{\perp}} \frac{\partial v_{y}}{\partial x}\right)\right]\right\}
\end{aligned}
$$


In these equations $c=\gamma T$ is the polytropic sound speed, $a_{y}$ and $a_{z}$ are the $y$-and $z$-components of the Alfvén speed, respectively, $a_{\perp}^{2}=a_{y}^{2}+a_{z}^{2}, \xi=a_{x}|| a_{x} \mid$, and

$$
\alpha_{1}=\sqrt{\frac{f^{2}-a_{x}^{2}}{f^{2}-s^{2}}}, \quad \alpha_{2}=\sqrt{\frac{f^{2}-c^{2}}{f^{2}-s^{2}}} .
$$

The normalization is conveniently chosen in order to prevent as much as possible the occurrence of singularities (Roe \& Balsara 1996). The explicit form of equation (A3) for the MHD equations, neglecting the terms that depend on the $y$-derivatives and the homogeneous terms eventually included in the matrix $\boldsymbol{C}$, is

$$
\begin{aligned}
\frac{\partial \rho}{\partial t} & =\frac{\rho}{c} \mathcal{L}_{0}+\rho\left[\frac{\alpha_{2}}{2 c}\left(\mathcal{L}_{s}^{+}+\mathcal{L}_{s}^{-}\right)-\frac{\alpha_{1}}{2 f}\left(\mathcal{L}_{f}^{+}+\mathcal{L}_{f}^{-}\right)\right] \\
\frac{\partial v_{x}}{\partial t} & =\frac{s \alpha_{2}}{2 c}\left(\mathcal{L}_{s}^{+}-\mathcal{L}_{s}^{-}\right)-\frac{\alpha_{1}}{2}\left(\mathcal{L}_{f}^{+}-\mathcal{L}_{f}^{-}\right), \\
\frac{\partial v_{y}}{\partial t} & =\frac{a_{z}}{2 a_{\perp}}\left(\mathcal{L}_{a}^{+}+\mathcal{L}_{a}^{-}\right)+\frac{a_{y}}{a_{\perp}}\left[\frac{\xi \alpha_{1}}{2}\left(\mathcal{L}_{s}^{+}-\mathcal{L}_{s}^{-}\right)+\frac{a_{x} \alpha_{2}}{2 f}\left(\mathcal{L}_{f}^{+}-\mathcal{L}_{f}^{-}\right)\right], \\
\frac{\partial v_{z}}{\partial t} & =-\frac{a_{y}}{2 a_{\perp}}\left(\mathcal{L}_{a}^{+}+\mathcal{L}_{a}^{-}\right)+\frac{a_{z}}{a_{\perp}}\left[\frac{\xi \alpha_{1}}{2}\left(\mathcal{L}_{s}^{+}-\mathcal{L}_{s}^{-}\right)+\frac{a_{x} \alpha_{2}}{2 f}\left(\mathcal{L}_{f}^{+}-\mathcal{L}_{f}^{-}\right)\right], \\
\frac{\partial B_{y}}{\partial t} & =-\xi \frac{\sqrt{4 \pi \rho} a_{z}}{2 a_{\perp}}\left(\mathcal{L}_{a}^{+}-\mathcal{L}_{a}^{-}\right)-\frac{\sqrt{4 \pi \rho} a_{y}}{a_{\perp}}\left[\frac{c \alpha_{1}}{2 f}\left(\mathcal{L}_{s}^{+}+\mathcal{L}_{s}^{-}\right)+\frac{\alpha_{2}}{2}\left(\mathcal{L}_{f}^{+}+\mathcal{L}_{f}^{-}\right)\right], \\
\frac{\partial B_{z}}{\partial t} & =\xi \frac{\sqrt{4 \pi \rho} a_{y}}{2 a_{\perp}}\left(\mathcal{L}_{a}^{+}-\mathcal{L}_{a}^{-}\right)-\frac{\sqrt{4 \pi \rho} a_{z}}{a_{\perp}}\left[\frac{c \alpha_{1}}{2 f}\left(\mathcal{L}_{s}^{+}+\mathcal{L}_{s}^{-}\right)+\frac{\alpha_{2}}{2}\left(\mathcal{L}_{f}^{+}+\mathcal{L}_{f}^{-}\right)\right], \\
\frac{\partial T}{\partial t} & =-\frac{T}{c} \mathcal{L}_{0}+T(\gamma-1)\left[\frac{\alpha_{2}}{2 c}\left(\mathcal{L}_{s}^{+}+\mathcal{L}_{s}^{-}\right)-\frac{\alpha_{1}}{2 f}\left(\mathcal{L}_{f}^{+}+\mathcal{L}_{f}^{-}\right)\right] .
\end{aligned}
$$

The characteristic form of the MHD equations can be used at the boundaries to know which information came from the computational domain and which information came from outside. If we put our attention to one boundary, for example, the left boundary, $x=0$, we note that characteristics whose eigenvalues are negative represent waves coming out from the computational domain, so extra information is not needed. On the other hand, characteristics with positive eigenvalues represent incoming waves, which have to be specified in order to have a well-posed problem. The total control on the incoming waves is obtained by choosing

$$
\mathcal{L}_{i}=f(y, t)-\boldsymbol{r}_{i}^{T} \boldsymbol{C} \frac{\partial \boldsymbol{U}}{\partial y}-\boldsymbol{r}_{i}^{T} \boldsymbol{D}
$$

where $f(y, t)$ is the triggering function of the selected wave we want to inject in the domain. This choice has the advantage of erasing perturbations that propagate along the boundary, because of the derivatives along the $y$-direction, which should not have their counterparts given by the derivative along the $x$-direction (Grappin et al. 2000). This can be seen when we want to maintain the equilibrium field, i.e., when we want $\partial \boldsymbol{U} / \partial t=0$. In that case, using equation (A2) for the characteristics corresponding to outgoing waves, we must set

$$
\mathcal{L}_{i}=\boldsymbol{r}_{i}^{T} \boldsymbol{C} \frac{\partial \boldsymbol{U}}{\partial y}-\boldsymbol{r}_{i}^{T} \boldsymbol{D}
$$

\section{REFERENCES}

Anastasiadis, A., \& Vlahos, L. 1991, A\&A, 245, 271

. 1994, ApJ, 428, 819

Aschwanden, M. J., De Pontieu, B., Schrijver, C. J., \& Title, A. M. 2002, Sol. Phys., 206, 99

Aschwanden, M. J., Fletcher, L., Schrijver, C. J., \& Alexander, D. 1999, ApJ, 520,880

Axford, W. I., McKenzie, J. F., Sukhorukova, G. V., Banaszkiewicz, M., Czechowski, A., \& Ratkiewicz, R. 1999, Space Sci. Rev., 87, 25

Bulanov, S. V., Echkina, E. Y., Inovenkov, I. N., Pegoraro, F., \& Pichushkin, V. V. 1999, Phys. Plasmas, 6, 802

Bulanov, S. V., Shasharina, S. G., \& Pegoraro, F. 1990, Plasma Phys. Controlled Fusion, 32, 377

Bulanov, S. V., \& Syrovatskii, S. I. 1980, Soviet J. Plasma Phys., 6, 661

Craig, I. J. D., \& McClymont, A. N. 1993, ApJ, 405, 207 1997, ApJ, 481, 996

Cranmer, S. R. 2002, Space Sci. Rev., 101, 229

Del Zanna, L., Schaekens, E., \& Velli, M. 2005, A\&A, 431, 1095
Del Zanna, L., Velli, M., \& Londrillo, P. 1998, A\&A, 330, L13 2001, A\&A, 367, 705

Einaudi, G. 1995, in Small-Scale Structures in Three-Dimensional Hydrodynamic and Magnetohydrodynamic Turbulence, ed. M. Meneguzzi, A. Pouquet, \& P.-L. Sulem (Berlin: Springer), 391

Einaudi, G., Chibbaro, S., Dahlburg, R. B., \& Velli, M. 2001, ApJ, 547, 1167

Einaudi, G., \& Velli, M. 1994, Space Sci. Rev., 68, 97

Grappin, R., Cavillier, E., \& Velli, M. 1997, A\&A, 322, 659

Grappin, R., Léorat, J., \& Buttighoffer, A. 2000, A\&A, 362, 342

Heyvaerts, J., \& Priest, E. R. 1983, A\&A, 117, 220

Jones, F. C., \& Ellison, D. C. 1991, Space Sci. Rev., 58, 259

Lele, S. K. 1992, J. Comput. Phys., 103, 16

Longcope, D. W., \& Sudan, R. N. 1992a, Phys. Rev. Lett., 68, 1706 . 1992b, ApJ, 384, 305

Malara, F., Primavera, L., \& Veltri, P. 1996, ApJ, 459, 347

Malara, F., \& Velli, M. 1994, in IAU Colloq. 144, Solar Coronal Structures, ed. V. Rusin, P. Heinzel, \& J.-C. Vial (Bratislava: VEDA), 443 
McLaughlin, J. A., \& Hood, A. W. 2004, A\&A, 420, 1129

Nakariakov, V. M., Ofman, L., DeLuca, E. E., Roberts, B., \& Davila, J. M. 1999, Science, 285, 862

Nakariakov, V. M., Roberts, B., \& Murawski, K. 1997, Sol. Phys., 175, 93

Poinsot, T. J., \& Lele, S. K. 1992, J. Comput. Phys., 101, 104

Priest, E., \& Forbes, T. 2000, Magnetic Reconnection (Cambridge: Cambridge Univ. Press)
Roe, P. L., \& Balsara, D. S. 1996, J. Appl. Math., 56, 57

Thompson, K. W. 1987, J. Comput. Phys., 68, 1

Tsiklauri, D., Arber, T. D., \& Nakariakov, V. M. 2001, A\&A, 379, 1098

Tsiklauri, D., Nakariakov, V. M., \& Arber, T. D. 2002, A\&A, 395, 285

Vanajakshi, T. C., Thompson, K. W., \& Black, D. C. 1989, J. Comput. Phys., 84,343

Vlahos, L. 1994, Space Sci. Rev., 68, 39 